Objectives: In the study, we evaluated the association between MeS, HR-QoL and QoL-related factors, such as depression, fatigue and physical activity. Methods: We conducted a cross-sectional study with retrospective evaluation of disease activity, damage and therapies cumulative dosage. MeS was defined according to International Federation of Diabetes (IFD) criteria. All patients were evaluated to explore MeS IFD criteria and other CVD risk factors (familiar history, lifestyle, smoking). SLE disease activity and damage were evaluated using SELENA-SLEDAI and SDI indices, respectively. Disease flares were retrospectively assessed by SFI index. HR-QoL was quantified by SF-36 instrument. We used Beck Depression Inventory (BDI) to assess depression and Facit-Fatigue to evaluate fatigue. Physical activity was quantified using International Physical Activity Questionnaire (IPAQ) and expressed according to categorical IPAQ total score. Patients also completed Pittsburgh Sleep Quality Index (PSQI) exploring sleep pathology.

Results: We enrolled 55 SLE patients (2 male and 53 female). Mean age was $45 \pm 12.5$. MeS prevalence was $23.6 \%$ and obesity (according to IFD definition) was recorded in $36.4 \%$ of patients. SLE patients with MeS presented reduced scores in SF-36 summary components MCS and PCS compared to patients without MeS ( $\mathrm{p} 0.002$ and p 0.04, respectively). The SF-36 individual components significantly decreased in MeS were the Mental Health, the Physical Rose and the Social Role ( $0.003, p 0.03, p 0.05$, respectively). In multiple linear regression the values of MCS was significantly associated only to obesity ( $p 0.01)$, while neither MeS it self nor any MeS components were associated to PCS values. $\mathrm{BDI}$ score was significantly higher and Facit-Fatigue score was reduced in SLE patients meeting MeS criteria compared to subjects without MeS $(p<0.0001, p$ 0.005 , respectively). A greater proportion of SLE patients with MeS presented almost mild depression ( $p$ 0.03). We found to be physically inactive, according to IPAQ score, the majority of SLE patients with MeS compared to patients without $\mathrm{MeS}(\mathrm{p}<0.0001)$. In multiple logistic regression, factors related to MeS were the Number of flares in the previous one year [OR $(95 \% \mathrm{Cl}) 13.7(1.7-107.8)]$, to have a $\mathrm{BDI}>13$ (to have almost mild depression) [OR $0.05(0.004-0.87)]$ and to be physically inactive (IPAQ=1) [OR $33.5(2.3-496.4)]$.

Conclusions: HR-QoL seems to be compromised in SLE patients with MeS, especially in mental components. Moreover, SLE patients with MeS often presented depression, are burdened by more severe fatigue and frequently are physically inactive. The presence of MeS in SLE was associated to the number of flare and, above all, to the physical inactivity, while not having depression seems exert a protective effect on MeS.

Disclosure of Interest: None declared

DOI: 10.1136/annrheumdis-2017-eular.6712

\section{THU0268 PREVALENCE AND FEATURES OF CELIAC DISEASE IN PATIENTS WITH SYSTEMIC AUTOIMMUNE DISEASES: RESULTS OF A LARGE MULTICENTER STUDY}

E. Bartoloni ${ }^{1}$, A. Alunno ${ }^{1}$, O. Bistoni ${ }^{1}$, L. Cavagna ${ }^{2}$, L. Nalotto ${ }^{3}$, C. Baldini ${ }^{4}$, R. Priori ${ }^{5}$, G. Picarelli ${ }^{5}$, C. Fischetti $^{6}$, F. Franceschini ${ }^{7}$, L. Quartuccio $^{8}$, F. Carubbi ${ }^{9}$, M. Fredi ${ }^{7}$, C. Montecucco ${ }^{2}$, A. Doria ${ }^{3}$, M. Mosca $^{4}$, G. Valesini ${ }^{10}$, A. Gabrielli ${ }^{6}$, S. De Vita ${ }^{11}$, R. Giacomelli ${ }^{9}$, R. Gerli ${ }^{1} .{ }^{1}$ Rheumatology Unit. University of Perugia, Perugia; ${ }^{2}$ Department of Rheumatology. University of Pavia, Pavia; ${ }^{3}$ Rheumatology Unit. University of Padova, Padova; ${ }^{4}$ Rheumatology Unit. University of Pisa, Pisa; ${ }^{5}$ Rheumatology Unity. Sapienza University, Roma; ${ }^{6}$ University 'Politecnica delle Marche', Ancona; ${ }^{7}$ Rheumatology and Clinical Immunology. Spedali Civili of Brescia, Brescia; ${ }^{8}$ Rheumatology Clinic. University of Udine, Udine; ${ }^{9}$ Division of Rheumatology. University of L'Aquila, L'Aquila; ${ }^{10}$ Rheumatology Unit. Sapienza University, Roma;

${ }^{11}$ Rheumatology Clinic. University of Udine, Udine, Italy

Background: Celiac disease (CD) is an inflammatory and immune-mediated gluten-dependent enteropathy occurring in genetically susceptible individuals. CD is recognized to affect between $0.6 \%$ and $1 \%$ of worldwide population, with wide regional differences. Disease clinical features are protean and highlight the systemic nature of the disease. In recent years, an increased prevalence of $C D$ has been also reported in patients with connective tissue diseases (CTDs). This association may be due to a shared genetic predisposition, to immunological mechanisms and/or exposure to a common triggering event. However, this observation remains controversial since data are usually based on descriptive case reports. Different methods of antibody detection and enrolled population sample size may contribute to result discordance. Moreover, CD diagnosis is often delayed because disease clinical spectrum may be atypical mimicking rheumatologic conditions and autoimmune disease itself may display typical symptoms of CD. Undoubtedly, awareness of CD occurrence in CTDs is important to prevent potential long-term systemic complications related to an unrecognized $\mathrm{CD}$ in these patients.

Objectives: To evaluate the prevalence of overt and subclinical $C D$ and features of the disease in a large cohort of patients with systemic lupus erythematosus (SLE), systemic sclerosis (SSc) and primary Sjögren's syndrome (pSS) with a multicenter prospective study involving 9 Italian Rheumatology Units.

Methods: Data from consecutive 580 SLE, 354 pSS and 524 SSc patients were collected. Disease-specific features were registered in patients with known CD. Remaining patients were tested for IgA transglutaminase (Eu-tTG ${ }^{\circledR}$ human IgA new, Eurospital S.p.A., Trieste). Anti-endomysium (EMA) IgA and IgG were tested in IgA tTG positive and borderline patients. Esophagogastroduodenoscopy with duodenal biopsy was proposed in IgA tTG+/EMA+, IgA tTG-/EMA+ and IgA tTG $\pm / E M A+$ patients.

Results: CD prevalence was 1,7\% in SLE, 7\% in pSS and $1,3 \%$ in SSc patients. Higher frequency of elevated liver enzymes was detected in SLE-CD and of herpetiform dermatitis in SSc-CD patients in comparison to the other groups $(p<0.05$ for both). Interestingly, pSS-CD and SSc-CD patients were younger and had a lower age at diagnosis in comparison to pSS and SSc without CD $(p<0.05$ for all). Of interest, higher prevalence of CD was detected in SSc patients with diffuse form in comparison to limited SSc $(86 \%$ vs $14 \%, p=0.002)$.

Conclusions: The results of the present large multicenter study confirm higher prevalence of $C D$ in CTD patients, especially in pSS. Screening of CD may be considered in younger patients with CTD and lower age at diagnosis. The strong association of CD with the diffuse type of SSc is of note and suggests that different, still unexplored, pathogenic mechanisms may characterize the two subsets of the disease.

Disclosure of Interest: None declared

DOI: 10.1136/annrheumdis-2017-eular.4388

\section{THU0269 ELECTROCARDIOGRAPHIC NONSPECIFIC ST-T ABNORMALITIES ARE ASSOCIATED WITH HIGHER MODIFIED FRAMINGHAM RISK SCORE IN SYSTEMIC LUPUS ERYTHEMATOSUS PATIENTS WITHOUT CLINICAL CARDIOVASCULAR DISEASE}

E. George, T. Perez-Recio, L. Geraldino-Pardilla. Department of Rheumatology, Columbia University College of Physicians \& Surgeons, New York, United States

Background: Cardiovascular disease (CVD) is a leading cause of mortality in systemic lupus erythematosus (SLE). Traditional CVD risk scores underperform in SLE. Interestingly, a high prevalence of nonspecific ST-T changes (NST-T) has been recently reported in lupus patients ${ }^{1,2}$. These electrocardiographic findings are known to increase the risk for myocardial infarction, coronary artery disease, CVD, and all-cause mortality ${ }^{3}$ in the general population, but in SLE this association remains unknown. Therefore, we sought to define the association of NST-T with the modified Framingham Risk Score (mFRS) as a surrogate outcome for CVD ${ }^{4}$. Objectives: To evaluate if NST-T are associated with higher mFRS in SLE patients without clinical cardiovascular disease.

Methods: Adult SLE patients without clinical CVD continuously seen at the Columbia University Lupus Center between April 2016 and January 2017, meeting 1997 American College of Rheumatology classification criteria for SLE were studied. Twelve-lead electrocardiogram (EKG), high sensitivity C-reactive Protein (hsCRP), demographics, disease-specific characteristics, medication use, and CVD risk factors were ascertained. Univariable and multivariable linear regression models were constructed to test the association of NST-T with the mFRS.

Results: Seventy-four lupus patients were studied (baseline characteristics in table 1). In univariable analysis, patients with NST-T had a significantly higher mFRS $(0.44, p=0.018)$. There were no confounders identified in the analysis. However after adjusting for variables associated with the mFRS: smoking, diabetes, hsCRP, Systemic Lupus International Collaborating Clinics/American College of Rheumatology Damage Index [SDI], aspirin use, this association remained statistically significant $(0.38, p=0.05)$. Image 1 shows the association of mFRS with NST-T vs no NST-T.

\begin{tabular}{lc}
\hline Age, years \pm SD & $39 \pm 13$ \\
Female, $\mathrm{n}(\%)$ & $67(90 \%)$ \\
Hispanic, $\mathrm{n}(\%)$ & $56(77 \%)$ \\
African American, $\mathrm{n}(\%)$ & $14(19 \%)$ \\
Body mass index & $30 \pm 8.3$ \\
Median Disease Duration, years (IQR) & $6.5(4-12)$ \\
Median SLEDAI (IQR) & $4(2-8)$ \\
Moderate-Severe Disease activity (SLEDAI $\geq 6), \mathrm{n}(\%)$ & $23(31 \%)$ \\
Median SDI, $\mathrm{n}(\%)$ & $0(0-1)$ \\
hsCRP median (IQR) & $2.4(1-6.5)$ \\
ESR, Median (IQR) & $37(23-51)$ \\
Anti-DNA, $\mathrm{n}(\%)$ & $44(60 \%)$ \\
Anti-SSA, $\mathrm{n}(\%)$ & $42(59 \%)$ \\
Anti-Smith, $\mathrm{n}(\%)$ & $21(30 \%)$ \\
Ever smoker, $\mathrm{n}(\%)$ & $16(22 \%)$ \\
Hypertension, $\mathrm{n}(\%)$ & $22(30 \%)$ \\
Diabetes, $\mathrm{n}(\%)$ & $6(8 \%)$ \\
mFRS, median (IQR) & $0.5(0-1.8)$ \\
NST-T abnormalities, $\mathrm{n}(\%)$ & $27(38 \%)$ \\
\hline
\end{tabular}

Conclusions: Non Specific ST-T changes are independently associated with a higher mFRS in SLE patients without clinical CVD.

References:

[1] Bourré-Tessier J, Urowitz MB, Pineau CA. et al. Electrocardiographic findings in systemic lupus erythematosus: data from an international inception cohort.Arthritis Care Res (Hoboken). 2015 Jan;67(1):128-35.

[2] Geraldino-Pardilla L,Gartshteyn Y, Piña P, et al.ECG non-specific ST-T and QTc abnormalities in patients with systemic lupus erythematosus compared with rheumatoid arthritis. Lupus Sci Med. 2016 Dec 16;3(1):e000168.

[3] Daviglus ML, Liao Y, Stamler J, et al. Association of Nonspecific Minor ST-T 


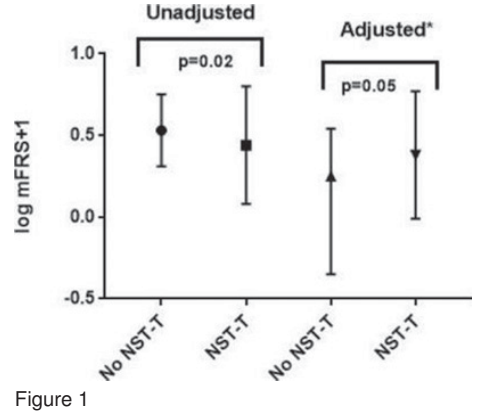

Abnormalities With Cardiovascular Mortality The Chicago Western Electric Study. JAMA. 1999;281(6):530-536.

[4] Urowitz MB, Ibañez D, Gladman DD,et al. Modified Framingham Risk Factor Score for Systemic Lupus Erythematosus. J Rheumatol. 2016 May;43(5):8759.

Disclosure of Interest: None declared

DOI: 10.1136/annrheumdis-2017-eular.4655

\section{THU0270 HOMOCYSTEINE, ANTIPHOSPHOLIPID ANTIBODIES AND RISK OF VASCULAR EVENTS IN PATIENTS WITH SYSTEMIC LUPUS ERYTHEMATOSUS}

G. Stojan, W. Fu, M. Petri. Rheumatology, Johns Hopkins University, Baltimore, United States

Background: SLE patients have higher plasma total homocysteine concentrations compared to healthy controls. Hyperhomocysteinemia in SLE is a potentially modifiable, independent risk factor for stroke and thrombotic events, hypertension, and coronary artery calcification.

Objectives: We investigated the association of homocysteine levels with the presence of antiphospholipid antibodies as well as the potentially additive thrombotic risk in patients with antiphospholipid antibodies who have hyperhomocysteinemia. Methods: To analyze the association between hyperhomocysteinemia and the presence of antiphospholipid antibodies in SLE, 844 patients with homocysteine measurements were included in the analysis. 237 patients had at least one measurement over $15 \mathrm{umol} / \mathrm{L}$. Patients were followed quarterly after cohort entry. The association of hyperhomocysteinemia with antiphospholipid antibodies is detailed in Table 1.

Table 1. Homocysteine and antiphospholipid antibody positivity

\begin{tabular}{lcccccc}
\hline & $\begin{array}{c}\text { Homocysteine } \\
\text { (umol/L) }\end{array}$ & $\begin{array}{c}\text { Odd ratios } \\
(95 \% \mathrm{Cl})\end{array}$ & \begin{tabular}{c} 
P value \\
\cline { 2 - 8 }
\end{tabular} & $\begin{array}{c}\text { Adj. Odd ratios } \\
(95 \% \mathrm{Cl})\end{array}$ & $\begin{array}{c}\text { Adj. } \\
\text { P value }\end{array}$ \\
\hline Anti-cardiolipin & $43.24)$ & 57.62 & $0.56(0.37,0.84)$ & 0.0049 & $0.54(0.36,0.81)$ & 0.0033 \\
Anti-B2 Glycoprotein & 16.98 & 29.69 & $0.48(0.28,0.82)$ & 0.0076 & $0.46(0.27,0.8)$ & 0.0054 \\
Lupus anticoagulant & 19.82 & 29.29 & $0.6(0.36,0.98)$ & 0.0404 & $0.54(0.33,0.91)$ & 0.0190 \\
\hline
\end{tabular}

To analyze the prevalence of vascular events among SLE patients with antiphospholipid antibodies based on homocysteine levels, 571 patients with positive antiphospholipid antibodies and at least one homocysteine measurement were included in the analysis. There were 166 patients with at least one homocysteine measurement over $15 \mathrm{umol} / \mathrm{L}$.

The lupus anticoagulant was assessed by dRVVT with mixing studies and confirmatory tests. Anticardiolipin and anti-beta2 glycoprotein 1 were measured by ELISA (INOVA). Vascular events were defined as stroke, myocardial infarction, digital gangrene, and deep vein thrombosis.

Results:

Table 2. Prevalence of vascular events among SLE patients with antiphospholipid antibodies based on homocysteine levels

\begin{tabular}{lccc}
\hline & Abnormal Homocysteine $>15$ umol/L & Normal Homocysteine & P value \\
& $\mathrm{N}(\%)$ & $\mathrm{N}(\%)$ & \\
\hline Superficial Thrombosis & $4(2.41)$ & $12(2.96)$ & 0.7176 \\
Deep vein thrombosis & $37(22.29)$ & $58(14.36)$ & 0.0257 \\
Stroke & $17(10.24)$ & $30(7.41)$ & 0.3139 \\
Myocardial infarction & $13(7.83)$ & $11(2.72)$ & 0.0099 \\
Digital Gangrene & $4(2.41)$ & $8(1.98)$ & 0.7523 \\
\hline
\end{tabular}

Conclusions: SLE patients with elevated homocysteine were less likely $(p<0.05)$ to have any of the antiphospholipid antibodies. Among patients with SLE who have antiphospholipid antibodies, elevated homocysteine is associated with a significantly higher prevalence of myocardial infarction and deep vein thrombosis $(\mathrm{p}<0.05)$.

Disclosure of Interest: None declared

DOI: 10.1136/annrheumdis-2017-eular.6705

\section{THU0271 THE PERFORMANCE OF DIFFERENT CLASSIFICATION CRITERIA IN PATIENTS WITH PRIMARY SJOGREN'S SYNDROME AND ANALYSIS OF THEIR CONTRIBUTION FOR DEFINITIVE DIAGNOSIS, WHEN EITHER CRITERIA USED ALONE OR IN COMBINATION}

Z. Kosuva Ozturk ${ }^{1}$, G. Kenar ${ }^{2}$, H. Yarkan Tugsal ${ }^{2}$, B. Zengin ${ }^{2}$, G. Can ${ }^{2}$, F. Onen ${ }^{2}$, M. Birlik ${ }^{2} .{ }^{1}$ Internal Medicine; ${ }^{2}$ Rheumatology, Dokuz Eylul University School of Medicine, Izmir, Turkey

Background: Many classification criteria sets have been proposed for primary Sjögren's Syndrome (pSS),consisting of variable features of the disease.Despite increasing number of criteria sets, expert opinion is still keeping its importance for the diagnosis of pSS.

Objectives: We aimed to compare the performance of 3 classification criteria sets for pSS, to determine the agreement between each other and expert opinion and to investigate diagnostic contribution with any combined use of these criteria.For those assessments, American-European Consensus Criteria (AECG), American Collage of Rheum (ACR)/Sjögren Int. Clinical Alliance (SICCA) criteria and L.S. Martin et al. criteria named Mathematical model (M.model) had chosen. The M.model is a criteria set known to be noninvasive and proposed as highly specific. Methods: 86 patients (F: $96 \%$, mean age: $51.7 \pm 11.8$ ) following up in our clinic with the diagnosis of pSS were enrolled to the study.Expert opinion had been taken as gold standard for pSS diagnosis. Patients were questioned for eye/mouth dryness. Antinuclear antibody (ANA), complement (C3, C4), anti-Ro and anti-La, serum protein electrophoresis, rheumatoid factor (RF), break-up time (BUT) and Schirmer, minor salivary gland biopsy results were analysed cautiously. The aforementioned criteria sets, were implemented to assess the classification. The percentage of exact agreement and Kappa test was calculated.

Results: Number of patients classified as pSS according to ACR/SICCA, AECG and M.model criteria were $75(87 \%), 63(73 \%)$ and $58(67 \%)$ respectively. 9 patients (10\%) did not fulfill any of these 3 criteria but they were diagnosed as pSS according to expert opinion (Image 1). The Kappa test was moderate between the ACR/SICCA and AECG criteria and slightly low between the M.model with the $A E C G$ and ACR/SICCA criteria (Table 1). According to our proposed combination model, number of patients classified as pSS, either AECG or M.model was 74(86\%); either AECG or ACR/SICCA model was 76(88\%); either ACR/SICCA or M.model was $77(89.5 \%)$ (Table 2). In the triple combination of our model, if all 3 criteria used concomitantly, only $46(51 \%)$ of patients fulfill the criteria simultaneously; however if any of 3 criteria sets used 77 (89.5\%) patients fulfill as pSS.

\begin{tabular}{|c|c|c|c|c|}
\hline & AECG & ACR/SICCA & \multicolumn{2}{|c|}{ M.MODEL } \\
\hline AECG & & $\% 83 \mathrm{~K}: 0.50$ & \multirow{2}{*}{\multicolumn{2}{|c|}{$\% 68 \mathrm{~K}: 0.25$}} \\
\hline ACR/SICCA & $\% 83 \mathrm{~K}: 0.50$ & & & \\
\hline M.MODEL & $\% 68 \mathrm{~K}: 0.25$ & $\% 75 \mathrm{~K}: 0.34$ & \multicolumn{2}{|c|}{$\% 75 \mathrm{~K}: 0.34$} \\
\hline \multicolumn{5}{|l|}{ Table 2} \\
\hline & & Classsification criteria & (n) & $(\%)$ \\
\hline \multirow{3}{*}{\multicolumn{2}{|c|}{ According to 1 criteria }} & ACR/SICCA & 75 & 87,2 \\
\hline & & AECG & 63 & 73,2 \\
\hline & & M. model & 58 & 67,4 \\
\hline \multirow{3}{*}{\multicolumn{2}{|c|}{ According to either of 2 criteria }} & ACR/SICCA or M.model & 77 & 89,5 \\
\hline & & AECG or ACR/SICCA & 76 & 88,3 \\
\hline & & AECG or M. model & 74 & 86 \\
\hline \multirow{2}{*}{\multicolumn{2}{|c|}{ According to either of 3 criteria }} & AECG or ACR/SICCA or M.model & 77 & 89,5 \\
\hline & & AECG + ACR/SICCA + M.model & 46 & 51.6 \\
\hline
\end{tabular}

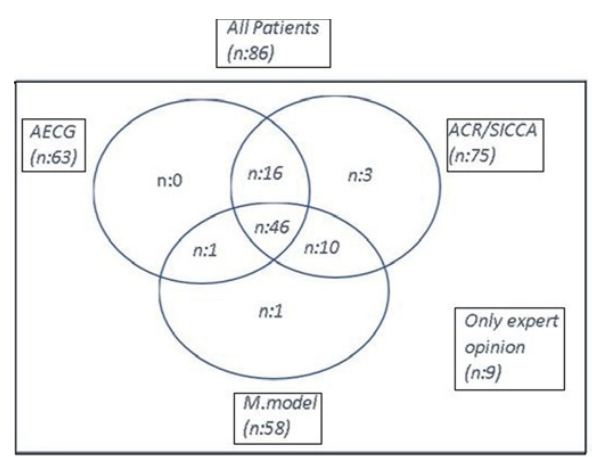

Conclusions: In this study, ACR/SICCA classification criteria for pSS was found the most compatible criteria set with expert opinion, when it used alone. The combination of either ACR/SICCA or M.model was found to be the most sensitive binary combination for classification with similar results as triple combination. This study showed that a small group of patients could not be classified as pSS even if the criteria sets were used with concomitant combinations. It was determined that experienced specialists were largely need to recognize and distinguish these patients.

Disclosure of Interest: None declared

DOI: 10.1136/annrheumdis-2017-eular.6074 